\title{
Respiratory impairment and COPD hospitalisation in older persons: a competing risk analysis
}

\author{
Carlos A. Vaz Fragoso*,\#, John Concato*,", Gail McAvay\#, \\ Peter H. Van Ness ${ }^{\#}$ and Thomas M. Gill
}

ABSTRACT: The aim of the present study was to evaluate, among older persons, the association between respiratory impairment and hospitalisation for chronic obstructive pulmonary disease (COPD), based on spirometric Z-scores, i.e. the LMS (lambda, mu, sigma) method, and a competing risk approach.

Using data on 3,563 white participants aged 65-80 yrs (from the Cardiovascular Health Study) we evaluated the association of LMS-defined respiratory impairment with the incident of COPD hospitalisation and the competing outcome of death without COPD hospitalisation, over a 5-yr period. Respiratory impairment included airflow limitation (mild, moderate or severe) and restrictive pattern.

Over a 5-yr period, 276 (7.7\%) participants had a COPD hospitalisation incident, whereas 296 (8.3\%) died without COPD hospitalisation. The risk of COPD hospitalisation was elevated more than two-fold in LMS-defined mild and moderate airflow limitation and restrictive pattern (adjusted HR (95\% Cl): 2.25 (1.25-4.05), 2.54 (1.53- 4.22) and 2.65 (1.82-3.86), respectively), and more than eight-fold in LMS-defined severe airflow limitation (adjusted HR (95\% Cl) 8.33 (6.24-11.12)). Conversely, only LMS-defined restrictive-pattern was associated with the competing outcome of death without COPD hospitalisation (adjusted HR (95\% CI) 1.68 (1.22-2.32)).

In older white persons, LMS-defined respiratory impairment is strongly associated with an increased risk of COPD hospitalisation. These results support the LMS method as a basis for defining respiratory impairment in older persons.

KEYWORDS: Chronic obstructive pulmonary disease hospitalisation, lambda, mu, sigma, spirometry

$\mathrm{H}$ ospitalisation for chronic obstructive pulmonary disease (COPD) has substantial clinical and societal importance, given the high rates of associated morbidity, mortality and healthcare costs, particularly in older persons $[1,2]$. Prior work, based on data from the Cardiovascular Health Study (CHS), has shown that spirometric respiratory impairment (airflow limitation and restrictive pattern), defined on the basis of criteria published by the Global Initiative for Chronic Obstructive Lung Disease (GOLD) [3], is strongly associated with the outcome of COPD hospitalisation [4]. However, the validity of these results is uncertain due to the three reasons discussed below.

First, the GOLD threshold for distinguishing normal spirometry from respiratory impairment is based on a fixed ratio of forced expiratory volume in 1s (FEV1) to forced vital capacity (FVC) of $0.70[3,4]$. Because ageing is associated with increasing rigidity of the chest wall and decreasing elastic recoil of the lung, an FEV1/FVC $<0.70$ frequently occurs in otherwise healthy neversmokers who are $\geqslant 65$ yrs [5-10].

Secondly, to establish restrictive pattern and to stage airflow limitation, respectively, the GOLD criteria expresses the FVC and FEV1 as per cent predicted (\% pred) $[3,4]$. Because ageing is also associated with increased variability in spirometric performance, increased disparity between $\%$ pred values and the lower limit of normal (LLN) is found among older persons [6-8, 11, 12].

Thirdly, Kaplan-Meier survival curves and standard Cox models were used exclusively to evaluate the association between GOLD-defined respiratory impairment and COPD hospitalisation [4]. Because the competing risk of death was not explicitly considered, traditional analytical methods, such as Kaplan-Meier and Cox methods, may introduce bias in an analysis of time to

\section{AFFILIATIONS}

*Veterans Affairs Clinical Epidemiology Research Center, West Haven, CT, and

\#Dept of Internal Medicine, Yale University School of Medicine, New Haven, CT, USA.

\section{CORRESPONDENCE}

C.A. Vaz Fragoso

Clinical Epidemiology Research

Center

VA Connecticut Healthcare System 950 Campbell Ave

Mailcode 151B

West Haven

CT

USA

E-mail: carlos.fragoso@yale.edu

Received:

July 272011

Accepted after revision:

Nov 092011

First published online:

Jan 202012 
COPD hospitalisation, as older persons frequently die without experiencing the outcome of interest, especially during an extended period of follow-up [13-15].

To address the above concerns, we set out in the current study to evaluate the association between a novel strategy for establishing spirometric respiratory impairment, termed LMS (lambda, mu, sigma) [6, 7], and the incident of COPD hospitalisation in older persons over a follow-up period of 5 yrs, using a competing risk analysis [13-15]. The LMS method calculates spirometric Z-scores that account for agerelated changes in pulmonary function, including variability in spirometric performance and skewness of reference data [6, 7]. Importantly, in the more informative competing risk analysis, we also included the outcome of death without COPD hospitalisation and used both Cox cause-specific and subdistribution hazards models [13-15]. Whereas cause-specific models evaluate relative risk, subdistribution models evaluate the cumulative burden of an outcome [13-15]. As a secondary aim, we compared the 5-yr cumulative incidence probabilities of COPD hospitalisation among participants who had discordant designations for respiratory impairment on the basis of the LMS and GOLD criteria.

\section{MATERIALS AND METHODS \\ Study population}

We used data from the CHS, with institutional review board approvals obtained at our respective institutions. CHS is a population-based, longitudinal study of 5,888 older Americans, with an age range of 65-100 yrs [16]. The study population was assembled in 1989-1990, as a random sample of Medicare beneficiaries in four US communities (participation rate of $57.3 \%$ ) [17].

Eligible participants for the present study were white, aged 65-80 yrs and had completed at least two American Thoracic Society (ATS) acceptable spirometric manoeuvers at baseline. Because reference values for the LMS method are currently unavailable for non-whites and those aged $>80 \mathrm{yrs}$, our analyses were limited to whites aged $65-80 \mathrm{yrs},[6,7]$. To focus on "irreversible" pathology as the principal comparison group for airflow limitation, participants with selfreported asthma were excluded. As per current convention, we did not exclude participants based on spirometric reproducibility criteria [18]. The final study sample included 3,563 participants.

\section{Spirometry}

In the CHS participants underwent spirometry during the baseline examination, according to contemporary ATS protocols [19]. For each participant, the measured FEV1/FVC was calculated from the largest set of FEV1 and FVC values that were recorded in any of the spirometric manoeuvres meeting ATS acceptability criteria [18-20].

Investigators have previously recommended that spirometric measures should be expressed as a Z-score, which converts a raw measurement on a test to a standardised score in units of standard deviations [11, 12]. More recently, a method for calculating spirometric Z-scores, termed LMS, has been proposed [6,7]. This strategy uses specific elements of a distribution, including: the median $(\mathrm{mu})$, which represents how spirometric measures change based on predictor variables; the coefficient-of-variation (sigma), which represents the spread of reference values and adjusting for non-uniform dispersion; and skewness (lambda), which represents the departure from normality [6, 7]. Clinically, Z-scores are used routinely to diagnose osteoporosis based on bone density testing and the LMS method is applied widely to growth charts $[6,21]$.

Based on measured values for each participant, percentile distribution of LMS-derived Z-scores (LMS-tiles) for FEV1, FVC and FEV1/FVC were calculated as: ((measured/predicted $\left.\mathrm{mu})^{\text {lambda }}-1\right) /($ lambda $\times$ sigma $)[6,7]$. Predicted values for the mu lambda and skewness were calculated from LMS equations $[6,7]$. A Z-score of -1.64 then defined the lower limit of normal as the 5th percentile of distribution (5 LMS-tile) [6, 7]. Using the 5 LMS-tile as a diagnostic threshold, participants were classified as having normal spirometry: FEV1/FVC and FVC $\geqslant 5$ LMS-tile; airflow limitation: FEV1/FVC $<5$ LMS-tile; and restrictive-pattern: FEV1/FVC $\geqslant 5$ LMS-tile but FVC $<5$ LMStile $[6,7,22-26]$.

The severity of LMS-defined airflow limitation was staged according to FEV1, based on prior work and expressed as a percentile distribution of LMS-tiles [25]. Specifically, a threelevel severity was applied with mild, moderate and severe airflow limitation, staged by FEV1 $\geqslant 5,0.5-4.9$ and $<0.5$ LMStile, respectively [25]. Prior work had shown that these FEV1 staging criteria are associated with respiratory symptoms and mortality [25].

Participants were also classified on the basis of GOLD criteria: normal spirometry was defined by FEV1/FVC $\geqslant 0.70$ and FVC $\geqslant 80 \%$ pred, airflow limitation by $\mathrm{FEV} 1 / \mathrm{FVC}<0.70$, and restrictive pattern by $\mathrm{FEV} 1 / \mathrm{FVC} \geqslant 0.70$ but $\mathrm{FVC}<80 \%$ pred [3]. The severity of GOLD-defined airflow limitation was staged according to FEV1 \% pred [3]. A three-level range of severity was applied (mild, moderate and severe), corresponding to published cut-off points of 80 and $50 \%$ pred [3]. The $\%$ pred for FVC and FEV1 were calculated as measured/ predicted mean $\times 100$, with predicted values derived from published multiple regression equations [27].

\section{Clinical measures}

Baseline characteristics included age, sex, body mass index (BMI), smoking history, chronic conditions, and health status [16]. The primary outcome of interest was incident COPD hospitalisation over a follow-up period of $5 \mathrm{yrs}$, defined as the first hospitalisation for which COPD was listed as a discharge diagnosis. To ascertain hospitalisation, medical records were obtained for all reported hospitalisations; Medicare utilisation files were also evaluated to determine potentially missed hospitalisations [16]. Up to 10 discharge codes from the International Classification of Diseases, Ninth Revision, Clinical Modification (ICD-9-CM) were abstracted for each hospitalisation [16]. A COPD hospitalisation was then identified by the ICD-9-CM codes of 491 (chronic bronchitis), 492 (emphysema) or 496 (chronic airway obstruction) [16, 28]. The competing outcome of death without COPD hospitalisation was based on reviews of obituaries, medical records and death certificates [16]. Vital status and (where appropriate) hospitalisation diagnoses were available on all study participants. 


\section{Statistical analysis}

Baseline characteristics were summarised, overall, and by LMS-defined spirometric categories, as mean values accompanied by standard deviations or as counts accompanied by percentages. For significance testing of differences between the respiratory impairment groups and the normal spirometry group, $\mathrm{p}$-values were adjusted for multiple comparisons using the false discovery rate procedure [29].

The associations of LMS-defined respiratory impairment with incident COPD hospitalisation were evaluated using competing risk analysis, with the competing outcome being death without COPD hospitalisation [13-15]. Frequency distributions for incident COPD hospitalisation and death without COPD hospitalisation were calculated over the course of $5 \mathrm{yrs}$, with results stratified by baseline spirometric category. As a measure of risk over time, Cox cause-specific hazards models were used to evaluate the associations of LMS-defined airflow limitation (mild, moderate and severe) and restrictive pattern with the outcomes of incident COPD hospitalisation and death without COPD hospitalisation. For each outcome, the reference group was defined by normal spirometry.

In the model for COPD hospitalisation, participants were censored if they died or had completed 5-yr follow-up without experiencing a COPD hospitalisation. For the model of death without COPD hospitalisation, participants were censored if they were hospitalised for COPD or were alive at the completion of the 5-yr follow-up. Noninformative censoring assumes that participants who experience a competing outcome had the same probability of the primary outcome as did others who were at risk at the time of the competing outcome and had the same profile of covariates [13]

The three LMS stages of airflow limitation and the LMSdefined restrictive pattern were treated as nominal categories. For each Cox cause-specific regression model, goodness-of-fit was assessed by model-fitting procedures and the analysis of residuals. The proportional hazards assumption was tested by using interaction terms crossing the time-to-event outcome and each variable in the multivariable model; the terms were retained if $\mathrm{p}<0.05$ after adjusting for the multiplicity of comparisons. Higher-order effects were tested for the continuous covariates and included in the final model if they met a forward selection criterion of $\mathrm{p}<0.20$ [30].

As a cumulative measure of absolute risk, Fine and Gray models of subdistribution hazards [13-15] were used to estimate the cumulative incident probabilities of COPD hospitalisation and death without COPD hospitalisation over 5 yrs, according to the three-level LMS staging of airflow limitation and LMS-defined restrictive pattern. These models were stratified to allow each spirometric category to have its own baseline subdistribution hazard function.

Lastly, we compared the 5-yr cumulative incident probability of COPD hospitalisation among participants who had discordant designations for respiratory impairment on the basis of the LMS and GOLD criteria. Because all participants who had GOLD-defined normal spirometry also had LMS-defined normal spirometry, but not vice versa, the discordant designations occurred exclusively as GOLD-defined respiratory impairment but LMS-defined normal spirometry. We could not meaningfully evaluate hazard ratios (HR) for these discordant designations; however, because the reference groups defining normal spirometry for LMS and GOLD differ, and the reference groups serve as the basis for calculating HR. Accordingly, inferences made from a direct comparison of such HR would be flawed.

In both the Cox cause-specific and subdistribution hazards models, adjustments were made for age, height, sex, smoking history, BMI, number of chronic conditions and health status. Because only 20 participants had missing values for these covariates, complete case analyses were conducted for all regression modelling. SAS 9.2 was used in the analyses (SAS Institute, Inc., Cary, NC), with a $\mathrm{p}<0.05$ (two-sided) denoting statistical significance.

\section{RESULTS}

Table 1 provides the baseline characteristics of the study population. Among all participants, the mean age was $71.5 \mathrm{yrs}$; $2,054(57.6 \%)$ out of 3,563 were female; and 1,991 (55.9\%) out of 3,563 were former or current smokers. The mean BMI was $26.3 \mathrm{~kg} \cdot \mathrm{m}^{-2}$, and the mean number of chronic conditions was 1.0; fair-to-poor health status was reported by 728 (20.4\%) out of 3,563. LMS-defined respiratory impairment was present in $825(23.2 \%)$ out of 3,563, including $492(13.8 \%)$ out of 3,563 with airflow limitation and $333(9.3 \%)$ out of 3,563 with restrictive pattern. Smoking prevalence, particularly current smoking, and the number of chronic conditions were highest among participants with LMS-defined respiratory impairment (airflow limitation and restrictive pattern) and lowest among those with normal spirometry.

Table 2 shows the frequency distributions of incident COPD hospitalisation and the competing outcome of death without COPD hospitalisation over a 5-yr period and according to LMS-defined spirometric category. The frequency of COPD hospitalisation ranged from $104(3.8 \%)$ out of 2,738 in the normal spirometry group to $102(40.3 \%)$ out of 253 in the severe airflow limitation group. The frequency of death without COPD hospitalisation ranged from $210(7.7 \%)$ out of 2,738 in the normal spirometry group to 48 (14.4\%) out of 333 in the restrictive-pattern group.

Table 3 shows Cox cause-specific HRs for incident COPD hospitalisation and the competing outcome of death without COPD hospitalisation, over a 5 -yr period and according to LMS-defined spirometric category. Relative to normal spirometry, the risk of COPD hospitalisation was elevated more than two-fold in mild and moderate airflow limitation and restrictive pattern, with adjusted HR (95\% CI) of 2.25 (1.25$4.05), 2.54$ (1.53-4.23) and 2.65 (1.82-3.86), respectively, and more than eight-fold in severe airflow limitation, with an adjusted HR (95\% CI) of 8.33 (6.24-11.12). For the outcome of death without COPD hospitalisation, restrictive-pattern, relative to normal spirometry, showed an increased risk, with an adjusted HR (95\% CI) of 1.68 (1.22-2.32). In contrast, airflow limitation was not associated with an increased risk of death without COPD hospitalisation.

Figure 1 shows the 5-yr cumulative incidence probabilities of COPD hospitalisation and death without COPD hospitalisation, according to LMS-defined spirometric category. For the two competing outcomes, the baseline spirometric categories 
TABLE 1 Baseline characteristics of study participants

\begin{tabular}{|c|c|c|c|c|c|c|}
\hline \multirow[t]{2}{*}{ Characteristics } & \multirow[t]{2}{*}{ All } & \multicolumn{5}{|c|}{ LMS-defined spirometric category } \\
\hline & & Normal & \multicolumn{3}{|c|}{ Airflow limitation } & Restrictive Pattern \\
\hline Participants & 3563 & 2738 & 107 & 132 & 253 & 333 \\
\hline Age yrs & $71.5 \pm 4.1$ & $71.5 \pm 4.1$ & $71.4 \pm 4.6$ & $71.3 \pm 4.3$ & $71.4 \pm 4.0$ & $71.6 \pm 4.3$ \\
\hline \multicolumn{7}{|l|}{ Smoking status ${ }^{\#}$} \\
\hline Never & $1572(44.1)$ & $1366(49.9)$ & $22(20.6)$ & $19(14.4)$ & $40(15.8)$ & $125(37.5)$ \\
\hline Former & $1566(44.0)$ & $1146(41.9)$ & $54(50.5)$ & $72(54.5)$ & $145(57.3)$ & $149(44.7)$ \\
\hline Current & $425(11.9)$ & $226(8.3)$ & $31(29.0)$ & $41(31.1)$ & $68(26.9)$ & $59(17.7)$ \\
\hline$B M I \mathbf{k g} \cdot \mathrm{m}^{-2}$ & $26.3 \pm 3.9$ & $26.3 \pm 3.8$ & $24.5 \pm 3.7^{+}$ & $26.0 \pm 4.1$ & $25.7 \pm 3.9^{+}$ & $27.6 \pm 4.3^{+}$ \\
\hline Chronic conditions & $1.0 \pm 0.9$ & $0.9 \pm 0.9$ & $0.8 \pm 0.9$ & $1.1 \pm 0.9^{+}$ & $1.3 \pm 1.1^{+}$ & $1.3 \pm 1.1^{+}$ \\
\hline
\end{tabular}

Data are presented as $\mathrm{n}$, mean \pm SD or $\mathrm{n}(\%)$. LMS: lambda, mu, sigma; BMI: body mass index; COPD: chronic obstructive pulmonary disease. LMS-defined spirometry categories were established using forced expiratory volume in $1 \mathrm{~s}$ (FEV1) to forced expiratory volume (FVC) ratio and FVC, both expressed as LMS-tiles (percentile distribution of LMS-derived Z-scores), were used to define the categories of normal (FEV1/FVC and FVC $>5$ LMS-tile), aiflow limitation (FEV1/FVC $<0.5$ LMS-tile) mild (FEV1 $\geqslant 5$ LMS-tile), moderate (FEV1 0.5-4.9 LMS-tile) and severe $<0.5$ LMS-tile), and restrictive pattern (FEV1/FVC $\geqslant 5$ LMS-tile and FVC $<5$ LMS-tile). ${ }^{*}: p<0.05$, false discovery rate adjusted for, comparing smoking status for each subgroup of respiratory impairment (airflow limitation and restrictive pattern) to normal spirometry. $\because$ : included self-reported COPD (chronic bronchitis or emphysema). ${ }^{+}: \mathrm{p}<0.05$, false discovery rate adjusted for, comparing each subgroup of respiratory impairment (airflow limitation and restrictive-pattern) to normal spirometry.

yielded a distinct set of cumulative incidence probabilities. By year 5 , the severe airflow limitation group showed the highest probability of COPD hospitalisation (HR $(95 \% \mathrm{CI}) 0.27(0.22-$ $0.31)$ ), but the lowest probability of death without COPD hospitalisation (HR (95\% CI) 0.05 (0.03-0.08)). Restrictive pattern yielded similar probabilities for COPD hospitalisation and death without COPD hospitalisation (HR (95\% CI) 0.10 $(0.07-0.13)$ and $0.12(0.09-0.16)$, respectively).

Table 4 shows frequency distributions and 5-yr cumulative incidence probabilities of COPD hospitalisation for the discordant designations of GOLD-defined respiratory impairment but LMS-defined normal spirometry. Of the 2,738 participants who had normal spirometry by LMS, GOLD defined $946(34.6 \%)$ as having respiratory impairment, including $541(19.8 \%)$ and $240(8.8 \%)$ as mild and moderate airflow limitation, respectively, and $165(6.0 \%)$ as restrictive pattern. The discordant designations of respiratory impairment by GOLD relative to LMS yielded low probabilities of COPD hospitalisation, ranging from only 0.04 to 0.06 . These values are comparable to those among participants who had normal spirometry by GOLD (0.03) or LMS (0.04) (fig. 1).

TABLE 2 Frequency distributions of the primary outcome of incident chronic obstructive pulmonary disease (COPD) hospitalisation and the competing outcome of death without COPD hospitalisation over the course of 5 yrs and according to baseline LMS (lambda, mu, sigma)-defined spirometry category

\begin{tabular}{lccc}
\hline $\begin{array}{l}\text { Normal } \\
\text { Airflow limitation }\end{array} \quad 2738(76.8)^{\#}$ & $104(3.8)$ & $210(7.7)$ \\
$\quad$ Mild & $107(3.0)^{\#}$ & $13(12.1)$ & $9(8.4)$ \\
$\quad$ Moderate & $132(3.7)^{\#}$ & $18(13.6)$ & $12(9.1)$ \\
$\quad$ Severe & $253(7.1)^{\#}$ & $102(40.3)$ & $17(6.7)$ \\
Restrictive pattern & $333(9.3)^{\#}$ & $39(11.7)$ & $48(14.4)$ \\
Total & $3,563(100)^{\#}$ & $276(7.8)$ & $296(8.3)$ \\
\hline
\end{tabular}

Data are presented as $n$ (\% of row), unless otherwise stated. LMS-defined spirometry categories were established using forced expiratory volume in $1 \mathrm{~s}$ (FEV1) to forced volume capacity (FVC) ratio and FVC, both expressed as LMS-tiles (percentile distribution of LMS-derived Z-scores), normal (FEV1/FVC and FVC $>5$ LMS-tile), airflow limitation ( $F E V_{1} / F V C<0.5$ LMS-tile) mild (FEV1 $\geqslant 5$ LMS-tile), moderate (FEV1 0.5-4.9 LMS-tile) and severe $<0.5$ LMS-tile), and restrictive pattern (FEV1/FVC $\geqslant 5$ LMS-tile and FVC $<5$ LMS-tile). * : per cent of study population. 
TABLE 3 Cox cause-specific hazard ratios for the primary outcome of incident chronic obstructive pulmonary disease (COPD) hospitalisation and the competing outcome of death without COPD hospitalisation over the course of 5 yrs and according to baseline LMS (lamda, mu, sigma)-defined spirometry category\#

\section{Normal}

Airflow limitation

Mild

Moderate

Severe

Restrictive pattern
1.00

\author{
$2.25(1.25-4.05)$ \\ $2.54(1.53-4.23)$ \\ $8.33(6.24-11.1)$ \\ 2.65 (1.82-3.86)
}

\author{
$1.00(0.51-1.97)$ \\ $1.06(0.59-1.91)$ \\ $0.90(0.54-1.49)$ \\ $1.68(1.22-2.32)$
}

Data are presented as hazard ratio (95\% confidence interval). LMS-defined spirometry categories were established using forced expiratory volume in $1 \mathrm{~s}$ (FEV1) to forced volume capacity (FVC) ratio and FVC, both expressed as LMS-tiles (percentile distribution of LMS derived Z-scores), normal (FEV1/FVC and FVC $>5$ LMS-tile), airflow limitation ( $F E V_{1} / F V C<0.5$ LMS-tile) mild (FEV $1 \geqslant 5$ LMS-tile), moderate (FEV1 0.5-4.9 LMS-tile) and severe (FEV1 $<0.5$ LMS-tile), and restrictive pattern (FEV1/FVC $\geqslant 5$ LMS-tile and FVC $<5$ LMS-tile). "*: total number of participants 3,563. ": adjusted for age, height, sex, smoking, body mass index (BMI), BMI², number of chronic conditions, perceived health status and perceived health status by time interaction. In the model of COPD hospitalisation, 38 (7.7\%) of the 492 participants who had airflow limitation and $48(14.4 \%)$ of the 333 participants who had restrictive pattern were censored because they died without having a COPD hospitalisation; the remaining cases were censored at the end of follow-up, as described in the Material and Methods section. ${ }^{+}$: adjusted for age, age ${ }^{2}$, height, sex, smoking, BMI, number of chronic conditions, and perceived health status. In the model of death without COPD hospitalisation, $133(27.0 \%)$ of the 492 participants who had airflow limitation and 39 (11.7\%) of the 333 participants who had restrictive pattern were censored because they had a COPD hospitalisation; the remaining cases were censored at the end of follow-up, see Material and Methods section.

\section{DISCUSSION}

In a large sample of community-living, white, older persons, we found that LMS-defined respiratory impairment was present in nearly one-quarter of participants and conferred an increased relative risk and 5-yr cumulative incidence probability of COPD hospitaliation. Participants who had LMS-defined severe airflow limitation were especially vulnerable, having an eight-fold elevation in the risk of COPD hospitalisation and the highest 5-yr cumulative incidence probability of COPD hospitalisation (0.27). We also found that LMS-defined restrictive pattern, but not airflow limitation, increased the risk for the competing outcome of death without COPD hospitalisation. Lastly, in direct comparisons between LMS and GOLD criteria, we found that more than one-third of participants who had normal spirometry by LMS had respiratory impairment by GOLD, and that these participants had a low cumulative incidence probability of COPD hospitalisation over 5 yrs, ranging from only 0.04 to 0.06 , which was comparable to that among participants who had normal spirometry by GOLD alone (0.03). These results support the LMS method as a basis for defining spirometric respiratory impairment, particularly regarding the diagnosis and staging of COPD.

In the present study, the strong association between LMSdefined respiratory impairment and COPD hospitalisation can be explained by three factors. First, both LMS-defined airflow limitation and restrictive pattern were associated with a higher prevalence of cigarette smoking, especially current smoking. Smoking is the most potent risk factor for developing COPD [1], which is most often characterised by airflow limitation but may also present as restrictive pattern (air trapping, respiratory muscle weakness or smoking-related interstitial lung disease) [31-36]. In addition, current smoking substantially increases the risk of COPD hospitalisation [1, 37, 38]. Secondly, because study participants who had self-reported asthma were excluded, the LMS-defined airflow limitation is likely to have represented irreversible pathology, a defining feature of COPD [1]. Thirdly, because LMS-derived Z-scores account for the three elements of the distribution (median, coefficient-ofvariation and skewness), the respiratory impairment defined by LMS is more likely to represent clinically significant pathology, rather than age-related reductions in pulmonary function [6]. This supposition is supported by the strong and independent associations between LMS-defined respiratory impairment and other health outcomes, including respiratory symptoms, frailty and all-cause mortality, as shown in prior studies [22-26].

The differences observed in the present study regarding the association of LMS-defined airflow limitation and restrictive pattern with the competing outcome of death without COPD hospitalisation suggest potential differences in underlying pathophysiology. Because a reduced FVC (a diagnostic criterion for restrictive pattern) is a risk factor for coronary heart disease and sudden cardiac death, the strong association between LMS-defined restrictive pattern and death without COPD hospitalisation suggests the additional contribution of a cardiovascular process $[39,40]$. In particular, heart failure may have mediated the association between LMS-defined restrictive pattern and death without COPD hospitalisation in this population of older persons [41]. The lack of an association between LMS-defined airflow limitation and death without COPD hospitalisation is consistent with a primary respiratory process. Of note and as shown in table 3 , when death without COPD hospitalisation was evaluated, $27.0 \%$ of participants who had airflow limitation were censored because they had a COPD hospitalisation. Otherwise, if competing risks are not 

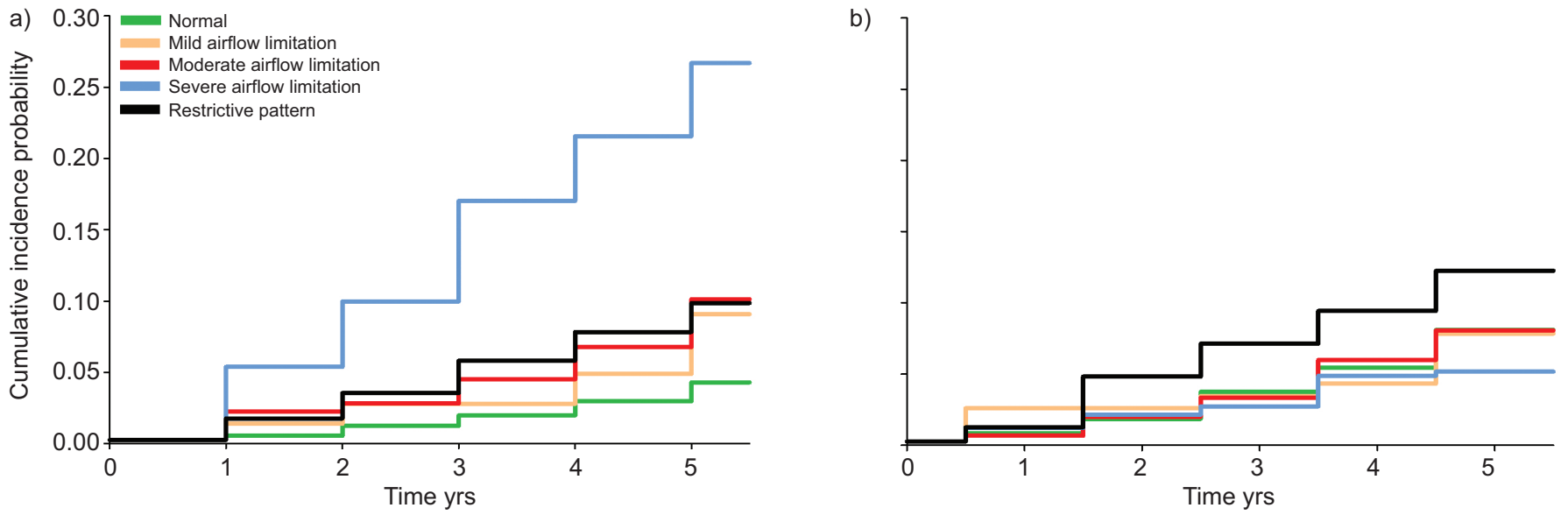

FIGURE 1. Cumulative incidence probability of a) chronic obstructive pulmonary disease (COPD) adjusted for age, height, sex, smoking history, body mass index (BMI), $\mathrm{BMI}^{2}$, number of chronic conditions and health status hospitalisation, and b) death without COPD hospitalisation, adjusted for age, age ${ }^{2}$, height, sex, smoking history, BMI, number of chronic conditions and health status over a 5-yr period, both according to baseline LMS (lambda, mu, sigma)-defined spirometry category. Forced expiratory volume in $1 \mathrm{~s}(\mathrm{FEV} 1)$ to forced expiratory volume (FVC) ratio and FVC, both expressed as LMS-tiles (percentile distribution of LMS-derived Z-scores), were used to define the categories of normal (FEV1/FVC and FVC $>5$ LMS-tile); airflow limitation (FEV1/FVC $<0.5$ LMS-tile) with mild (FEV1 $\geqslant 5$ LMS-tile), moderate (FEV1 $0.5-4.9$ LMS-tile) and severe (FEV1 $<0.5$ LMS-tile); and restrictive pattern ( $F E V_{1} / F V C \geqslant 5 L M S$-tile and FVC $<5$ LMS-tile). The cumulative incidence probability (95\% $\left.\mathrm{Cl}\right)$ for a and $\mathrm{b}$, respectively, for each LMS-defined spirometry category were: normal $0.04(0.036-0.05)$ and $0.08(0.07-0.09)$; airflow limitation mild $0.09(0.05-0.14)$ and 0.08 (0.03-0.12); airflow limitation moderate: $0.10(0.06-0.15)$ and 0.08 (0.04-0.12); airflow limitation severe: 0.27 (0.22-0.31) and 0.05 (0.03-0.08); and restrictive pattern: 0.10 (0.07-0.13) and 0.12 $(0.09-0.16)$

considered, we have shown previously that LMS-defined airflow limitation confers a comparable risk of death to that of restrictive pattern [24]. Future work should evaluate the factors that lead to death, including intervening illnesses resulting in hospitalisation [42], among older persons who have LMS-defined restrictive pattern and airflow limitation.

We found that more than one-third of participants who had normal spirometry defined by LMS had respiratory impairment as defined by GOLD, and that these participants had low probabilities of COPD hospitalisation, which were similar to that of participants who had normal spirometry by GOLD. These results support the view that respiratory impairment is over diagnosed by GOLD criteria. Defining respiratory impairment based on the LMS approach, rather than the GOLD approach, has a strong mathematical and clinical justification. Using a single diagnostic threshold set at a Z-score of -1.64 (LLN), the present study and prior work have shown that

\section{TABLE 4 Frequency distributions and 5-yr cumulative incidence probabilities of chronic obstructive pulmonary disease (COPD) hospitalisation for the discordant designation of Global Initiative for Chronic Obstructive Lung Disease (GOLD)-defined respiratory impairment but LMS (lambda, mu, sigma)-defined normal spirometry}

GOLD-defined spirometric category

LMS-defined normal spirometry

Participants n (\%)

Cumulative incidence probability $(95 \% \mathrm{Cl})$ of COPD hospitalisation ${ }^{+}$

\section{Normal \\ Airflow limitation \\ Mild \\ Moderate \\ Severe}

Restrictive pattern
$1792(65.5) \S$

$541(19.8)$

$240(8.8)$

$0(0)$

$165(6.0)$
$0.03(0.02-0.04)$

$0.06(0.04-0.07)$

$0.05(0.03-0.08)$

$0.04(0.01-0.08)$

LMS-defined normal spirometry was established by forced expiratory volume in $1 \mathrm{~s}(\mathrm{FEV} 1)$ /forced vital capacity $(\mathrm{FVC})$ and FVC, both $\geqslant 5$ LMS-tile. \#: the discordant designations for respiratory impairment occurred exclusively as GOLD abnormal but LMS normal, because all participants who had GOLD-defined normal spirometry also had LMS-defined normal spirometry, but not vice versa; ": GOLD-defined normal spirometry was established by FEV 1 to FVC ratio $\geqslant 0.70$ and FVC $\geqslant 80 \%$ predicted (\% pred was calculated by: (measured/predicted mean) $\times 100$ ); aiflow limitation was defined by FEV1/FVC $<0.70$, with mild as $F E V_{1} \geqslant 80 \%$ pred, moderate as $F E V 150-79 \%$ pred, and severe as $\mathrm{FEV} 1<50 \%$ pred; and restrictive pattern by $\mathrm{FEV} 1 / \mathrm{FVC} \geqslant 0.70$ but $\mathrm{FVC}<80 \%$ pred. ${ }^{+}$: adjusted for age, height, sex, smoking history, body mass

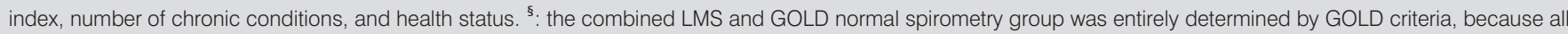
participants who had GOLD-defined normal spirometry also had LMS-defined normal spirometry, but not vice versa. 
LMS spirometric criteria yield plausible rates of respiratory impairment in ageing populations, and are associated with multiple adverse outcomes [22-26]. This has implications for clinical practice. Specifically, because it is less likely to misidentify normal ageing as respiratory impairment, the LMS approach may avoid inappropriate and harmful pharmacotherapy, as well as delays in the consideration of other diagnoses [22-26, 43-46].

Although the present study avoids specific problems found in prior reports, several potential limitations should be noted. First, LMS prediction equations have not yet been published for non-white subjects. Because racial differences exist in pulmonary function, our results may not be generalised to groups other than whites [6,47]. Secondly, we cannot confirm the specific pathophysiology that led to respiratory impairment. In addition to COPD, airflow limitation could have been due to bronchiectasis, given its increased prevalence in COPD, or to asthma, given that spirometry in CHS was not specifically obtained after a bronchodilator, that self-reported asthma (a key exclusion criterion) may have been underreported by participants, and that longstanding asthma may similarly lead to irreversible airflow limitation [48-52]. In addition to COPD and heart failure, other underlying causes for restrictive pattern may have also included thoracic kyphosis, nonCOPD related respiratory muscle weakness, and non-smoking related interstitial lung disease (usual interstitial pneumonia) [53-56]. Nonetheless, because LMS-defined airflow limitation and restrictive pattern were associated with an increased prevalence of smoking and risk of COPD hospitalisation, the predominant contributor to respiratory impairment was likely to be COPD.

To address these unanswered questions the association of LMS-defined respiratory impairment and COPD hospitalisations should be confirmed in subsequent studies that include participants from other racial and ethnic groups and that use postbronchodilator spirometry (we note that LMS-prediction equations will soon be available for non-whites subjects) [7]. Furthermore, to better evaluate the disease processes that underlie the association between respiratory impairment and COPD hospitalisations, future studies may need to include echocardiography, chest imaging, and more comprehensive tests of pulmonary function, including body plethysmography (e.g. to confirm restrictive lung disease) [57, 58], respiratory muscle pressures and diffusion capacity.

In summary, among community-living white older persons and in an analysis that explicitly addressed the competing risk of death, LMS-defined respiratory impairment significantly increased the relative risk and cumulative incidence probability of COPD hospitalisation. These results, together with previously established associations with respiratory symptoms, frailty, and all-cause mortality, provide strong support for the LMS method as a basis for defining respiratory impairment in ageing populations $[6,7,22-26]$.

\section{SUPPORT STATEMENT}

C.A. Vaz Fragoso is currently a recipient of a Career Development Award from the Department of Veterans Affairs and an R03 award from the National Institute on Aging (R03AG037051). J. Concato is supported by the Department of Veterans Affairs Cooperative Studies Program. M. Gill is the recipient of an NIA Midcareer Investigator
Award in Patient-Oriented Research (K24AG021507). The investigators retained full independence in the conduct of this research.

\section{STATEMENT OF INTEREST}

None declared.

\section{ACKNOWLEDGEMENTS}

C.A. Vaz Fragoso had full access to all of the data in the study and takes responsibility for the integrity of the data and the accuracy of the data analysis. All authors made substantial contributions to study concept and design, to data acquisition, analysis and interpretation, and to drafting the submitted article.

The Cardiovascular Health Study (CHS) was conducted and supported by the US National Heart, Lung, and Blood Institute (NHLBI) in collaboration with the CHS Study Investigators. This manuscript was prepared using a limited access dataset obtained from the NHLBI and does not necessarily reflect the opinions or views of the $\mathrm{CHS}$ or the NHLBI.

\section{REFERENCES}

1 Viegi G, Pistelli F, Sherrill DL, et al. Definition, epidemiology and natural history of COPD. Eur Respir J 2007; 30: 993-1013.

2 Albertson TE, Louie S, Chan AL. The diagnosis and treatment of elderly patients with acute exacerbation of chronic obstructive pulmonary disease and chronic bronchitis. J Am Geriatr Soc 2010; 58: 570-579.

3 Rabe KF, Hurd S, Anzueto A, et al. Global strategy for the diagnosis, management, and prevention of chronic obstructive pulmonary disease. GOLD executive summary. Am J Respir Crit Care Med 2007; 176: 532-555.

4 Mannino DM, Buist AS, Vollmer WM. Chronic obstructive pulmonary disease in the older adult: what defines abnormal lung function? Thorax 2007; 62: 237-241.

5 Meyer KC. Aging. Proc Am Thorac Soc 2005; 2: 433-439.

6 Stanojevic S, Wade A, Stocks J, et al. Reference ranges for spirometry across all ages a new approach. Am J Respir Crit Care Med 2008; 177: 253-260.

7 Lung Function in Growth and Aging. Download Software and Tools. www.lungfunction.org/growinglungs/software.html Date last updated: January 13, 2011. Date last accessed: June 20, 2011.

8 Quanjer PH, Enright PL, Miller MR, et al. The need to change the method for defining mild airway obstruction. Eur Respir J 2011; 37: $720-722$.

9 Celli BR, Halbert RJ, Nordyke RJ, et al. Airway obstruction in never smokers: results from the Third National Health and Nutrition Examination Survey. Am J Med 2005; 118: 1364-1372.

10 Vaz Fragoso CA, Concato J, McAvay G, et al. Chronic obstructive pulmonary disease in older persons: a comparison of two spirometric definitions. Respir Med 2010; 104: 1189-1196.

11 Miller MR, Pincock AC. Predicted values: how should we use them? Thorax 1988; 43: 265-267.

12 Quanjer PH, Tammeling GJ, Cotes JE, et al. Lung volumes and forced ventilatory flows Report Working Party Standardization of Lung Function Tests, European Community for Steel and Coal. Eur Respir J 1993; 6: Suppl. 16, 5-40.

13 Berry SD, Ngo L, Samelson EJ, et al. Competing risk of death: an important consideration in studies of older adults. J Am Geriatr Soc 2010; 58: 783-787.

14 Fine JP, Gray RJ. A proportional hazards model for the subdistribution of a competing risk. J Am Stat Assoc 1999; 94: 496-509.

15 Zhang X, Zhang M-J. SAS macros for estimation of direct adjusted cumulative incidence curves under proportional subdistribution hazards models. Comput Methods Programs Biomed 2011; 101: 87-93. 
16 Fried LP, Borhani NO, Enright P, et al. The cardiovascular health study: design and rationale. Ann Epidemiol 1991; 1: 263-276.

17 Tell GS, Fried LP, Hermanson B, et al. Recruitment of adults 65 years and older as participants in the Cardiovascular Health Study. Ann Epidemiol 1993; 3: 358-366.

18 Miller MR, Hankinson J, Brusasco V, et al. Standardisation of spirometry. Eur Respir J 2005; 26: 319-338.

19 Lung function testing: selection of reference values and interpretative strategies. American Thoracic Society. Am Rev Respir Dis 1991; 144: 1202-1218.

20 Pellegrino R, Viegi G, Brusasco V, et al. Interpretative strategies for lung function tests. Eur Respir J 2005; 26: 948-968.

21 Cummings SR, Bates D, Black DM. Clinical use of bone densitometry: scientific review. JAMA 2002; 288: 1889- 1897.

22 Vaz Fragoso CA, Concato J, McAvay G, et al. The ratio of the FEV1 to FVC as a basis for establishing chronic obstructive pulmonary disease. Am J Respir Crit Care Med 2010; 181: 446-451.

23 Vaz Fragoso CA, Gill TM, McAvay G, et al. Use of lambda-musigma-derived $\mathrm{Z}$ score for evaluating respiratory impairment in middle-aged persons. Respir Care 2011: 56; 1771-1777.

24 Vaz Fragoso CA, Gill TM, McAvay G, et al. Respiratory impairment and mortality in older persons: a novel spirometric approach. J Investig Med 2011; 59: 1089-1095.

25 Vaz Fragoso CA, Concato J, McAvay G, et al. Staging the severity of chronic obstructive pulmonary disease in older persons based on spirometric Z-scores. J Am Geriatr Soc 2011; 59: 1847-1854.

26 Vaz Fragoso CA, Enright PL, McAvay G, et al. Frailty and respiratory impairment in older persons. Am J Med 2012; 125: 79-86.

27 Hankinson JL, Odencrantz JR, Fedan KB. Spirometric reference values from a sample of the general US population. Am J Respir Crit Care Med 1999; 159: 179-187.

28 Lee TA, Wilke C, Joo M, et al. Outcomes associated with tiotropium use in patients with chronic obstructive pulmonary disease. Arch Intern Med 2009; 169: 1403-1410.

29 Benjamini Y, Hochberg Y. Controlling the false discovery rate: a practical and powerful approach to multiple testing. J R Stat Soc Series B Stat Methodol 1995; 51: 289-300.

30 Peduzzi P, Concato J, Feinstein AR, et al. Importance of events per independent variable in proportional hazards regression analysis. II. Accuracy and precision of regression estimates. J Clin Epidemiol 1995; 48: 1503-1510.

31 Washko GR, Hunninghake GM, Fernandez IE, et al. Lung volumes and emphysema in smokers with interstitial lung abnormalities. N Engl J Med 2011; 364: 897-906.

32 Vassallo R, Jensen EA, Colby TV, et al. The overlap between respiratory bronchiolitis and desquamative interstitial pneumonia in pulmonary Langerhans cell histiocytosis: high-resolution CT, histologic, and functional correlations. Chest 2003; 124: 1199-1205.

33 Cottin V, Le Pavec J, Prévot G, et al. Pulmonary hypertension in patients with combined pulmonary fibrosis and emphysema syndrome. Eur Respir J 2010; 35: 105-111.

34 Mannino DM, Holguin F, Pavlin BI, et al. Risk factors for prevalence of and mortality related to restriction on spirometry: findings from the first National Health and Nutrition Examination Survey and follow-up. Int J Tuberc Lung Dis 2005; 9: 613-621.

35 Dykstra BJ, Scanlon PD, Kester MM, et al. Lung volumes in 4,774 patients with obstructive lung disease. Chest 1999; 115: 68-74.

36 McKenzie DK, Butler JE, Gandevia SC. Respiratory muscle function and activation in chronic obstructive pulmonary disease. J Appl Physiol 2009; 107: 621-629.

37 Celli BR, MacNee W, Agusti A, et al. Standards for the diagnosis and treatment of patients with COPD: a summary of the ATS/ERS position paper. Eur Respir J 2004; 23: 932-946.
38 Burgel P-R, Nesme-Meyer P, Chanez P, et al. Cough and sputum production are associated with frequent exacerbations and hospitalizations in COPD subjects. Chest 2009; 135: 975-982.

39 Friedman GD, Klatsky AL, Siegelaub AB. Lung function and risk of myocardial infarction and sudden cardiac death. N Engl J Med 1976; 294: 1071-1075.

40 Lee HM, Le H, Lee BT, et al. Forced vital capacity paired with Framingham Risk Score for prediction of all-cause mortality. Eur Respir J 2010; 36: 1002-1006.

41 Hannink JD, van Helvoort HA, Dekhuijzen PN, et al. Heart failure and COPD: partners in crime? Respirology 2010; 15: 895-901.

42 Gill TM, Allore HG, Gahbauer EA, et al. Change in disability after hospitalization or restricted activity in older persons. JAMA 2010; 304: 1919-1928.

43 Singh S, Loke YK, Furberg CD. Inhaled anticholinergics and risk of major adverse cardiovascular events in patients with chronic obstructive pulmonary disease: a systematic review and metaanalysis. JAMA 2008; 300: 1439-1450.

44 Drummond MB, Dasenbrook EC, Pitz MW, et al. Inhaled corticosteroids in patients with stable chronic obstructive pulmonary disease: a systematic review and meta-analysis. JAMA 2008; 300: 2407-2416.

45 Stephenson A, Seitz D, Bell CM, et al. Inhaled anticholinergic drug therapy and the risk of acute urinary retention in chronic obstructive pulmonary disease: a population-based study. Arch Intern Med 2011; 171: 914-920.

46 Taylor DR. The beta-agonist saga and its clinical relevance: on and on it goes. Am J Respir Crit Care Med 2009; 179: 976-978.

47 Dransfield MT, Bailey WC. COPD: racial disparities in susceptibility, treatment, and outcomes. Clin Chest Med 2006; 27: 463-471.

48 Enright PL, McClelland RL, Newman AB, et al. Underdiagnosis and undertreatment of asthma in the elderly. Chest 1999; 116: 603-613.

49 Patel IS, Vlahos I, Wilkinson TMA, et al. Bronchiectasis, exacerbation indices, and inflammation in chronic obstructive pulmonary disease. Am J Respir Crit Care Med 2004; 170: 400-407.

50 Seitz AE, Olivier KN, Steiner CA, et al. Trends and burden of bronchiectasis-associated hospitalizations in the United States, 1993-2006. Chest 2010; 138: 944-949.

51 Behrendt CE. Mild and moderate-to-severe COPD in nonsmokers: distinct demographic profiles. Chest 2005; 128: 1239-1244.

52 Slavin RG, Haselkorn T, Lee JH, et al. Asthma in older adults: observations from the epidemiology and natural history of asthma: outcomes and treatment regimens (TENOR) study. Ann Allergy Asthma Immunol 2006; 96: 406-414.

53 Di Bari M, Chiarlone M, Matteuzzi D, et al. Thoracic kyphosis and ventilatory dysfunction in unselected older persons: an epidemiological study in Dicomano, Italy. J Am Geriatr Soc 2004; 52: 909-915.

54 American Thoracic Society, European Respiratory Society. American Thoracic Society/European Respiratory Society international multidisciplinary consensus classification of the idiopathic interstitial pneumonias. Am J Respir Crit Care Med 2002; 165: 277-304.

55 Georgiopoulou VV, Kalogeropoulos AP, Psaty BM, et al. Lung function and risk for heart failure among older adults: the Health ABC Study. Am J Med 2011; 124: 334-341.

56 Chan ED, Welsh CH. Geriatric respiratory medicine. Chest 1998; 114: 1704-1733.

57 Glady CA, Aaron SD, Lunau M, et al. Spirometry-based algorithm to direct lung function testing in the pulmonary function laboratory. Chest 2003; 123: 1939-1946.

58 Aaron SD, Dales RE, Cardinal P. How accurate is spirometry at predicting restrictive pulmonary impairment? Chest 1999; 115: 869-873. 